\title{
Establecimiento de cultivos bioenergéticos como fuente de energías alternativas, mediante el desarrollo de materiales de siembra en tres sitios de Costa Rica
}

\section{Establishment of bioenergy crops as a source of alternative energy, through the development of planting options in three sites in Costa Rica}

Elizabeth Arnáez-Serrano', lleana Moreira-González², Franklin Herrera-Murillo ${ }^{3}$, Guillermo Vargas-Hernández ${ }^{4}$, Emanuel Araya Valverde ${ }^{5}$, Elemer Briceño-Elizondo ${ }^{6}$, Katherine Sánchez-Zúñiga ${ }^{7}$

Arnáez-Serrano, E; Moreira-González, I; Herrera Murillo, F; Vargas-Hernández, G;

Araya-Valverde, E; Briceño-Elizondo, E; Sánchez-Zúñiga, K. Establecimiento de cultivos bioenergéticos como fuente de energías alternativas, mediante el desarrollo de materiales de siembra en tres sitios de Costa Rica. Tecnolog ía en Marcha. Vol. 32, Especial. VIII Encuentro de Investigación y Extensión. Abril 2019. Pág 25-34.

DOI: https://doi.org/10.18845/tm.v32i6.4225

Bióloga. Escuela de Biología. Instituto Tecnológico de Costa Rica. Costa Rica. Correo electrónico: earnaez @tec.ac.cr

2 Bióloga. Escuela de Biología. Instituto Tecnológico de Costa Rica. Costa Rica. Correo electrónico: ilea2757@gmail.com

3 Ingeniero Agrónomo. Facultad de Agronomía. Universidad de Costa Rica. Costa Rica. Correo electrónico: franklin.herrera@ucr.ac.cr

4 Ingeniero Agrónomo. Facultad de Agronomía. Universidad de Costa Rica. Costa Rica. Correo electrónico: guillermo.vargas@ucr.ac.cr

5 Ingeniero en Biotecnología. Escuela de Biología Instituto Tecnológico de Costa Rica y CENIBiot. Costa Rica. Correo electrónico: emaraya@gmail.com

6 Ingeniero Forestal. Escuela de Biología Instituto Tecnológico de Costa Rica. Costa Rica. Correo electrónico: ebriceno@tec.ac.cr

7 Ingeniera en Biotecnología. Escuela de Biología Instituto Tecnológico de Costa Rica. Costa Rica. Correo electrónico: katsanchez@tec.ac.cr 


\title{
Palabras clave
}

Cultivos bioenergéticos, Jatropha curcas, Ricinus comunis, Acrocomia aculeata.

\section{Resumen}

En Costa Rica, como una alternativa al uso de hidrocarburos, se ha venido buscando el empleo de cultivos bionergéticos de los cuales se pueda hacer un mayor aprovechamiento de la biomasa producida y la obtención de aceite. Durante los últimos ocho años, un grupo de interdisciplinario de investigadores de las universidades estatales de Costa Rica, han venido realizando estudios sistemáticos sobre el cultivo de Jatropha curcas (Tempate) para su uso comercial como fuente de aceite para producir biodiesel y a la vez en el 2017 inició el trabajo con otras especies con potencial bioenergético en el país como higuerilla (Ricinus comunis) y coyol (Acrocomia aculeata), entre otras. La investigación que se llevó a cabo, cubre tres objetivos donde el primero tiene que ver con el establecimiento de cultivos de estas especies en diferentes zonas bioclimáticas del país, así como ensayos sistemáticos alternativos con (tempate, higuerilla y coyol), combinándolos con plantas que se utilizan para alimento humano y animal. El segundo objetivo está relacionado con ofrecer a largo plazo variedades más productivas y menos tóxicas del tempate (Jatropha curcas). Y el último objetivo tiene que ver con el proceso de transferencia de información y resultados obtenidos. La información obtenida permitirá ofrecer opciones a los agricultores que les ayude a minimizar el impacto del cambio climático en su actividad y contribuir a las metas que Costa Rica se ha propuesto para reducir la emisión de contaminantes al ambiente y mejorar la calidad de vida de la población costarricense.

\section{Keywords}

Bioenergy crops, Jatropha curcas, Ricinus comunis, Acrocomia aculeata.

\begin{abstract}
In Costa Rica, an alternative to the use of fossil fuels is the use of plants with energetic potential; be this the direct biomass produced or the acquisition of fine oils. During the last eight years, a group of interdisciplinary researchers from the state universities of Costa Rica, have been conducting systematic studies on the cultivation of Jatropha curcas (Tempate) for commercial use as a source of oil to produce biodiesel; parallel since 2017 work with other species with bioenergy potential started, using castor bean (Ricinus comunis) and coyol (Acrocomia aculeata), among others. The research covers three objectives, first to establish crops of these species in different bioclimatic zones around the country, along with alternative systematic trials with (tempate, castor and coyol), in combination with food crops and animal feed. The second objective is focused on offering more productive and less toxic varieties of tempate (Jatropha curcas). And the last objective deals with technology and information transfer to the productive sector. The information obtained will allow new options to farmers and aid them to minimize the impact of climate change on their activities, as well as to contribute to the goals Costa Rica has proposed to reduce the emission and improve the quality of life of the Costa Rican population
\end{abstract}




\section{Introducción}

Los biocombustibles como fuente de energía poseen grandes beneficios al compararlos con los combustibles fósiles tradicionales, pues pueden generar energía carbono neutral [1]. A nivel mundial, los cultivos para biocombustibles consideran un amplio espectro de especies, pero presentan sus diferencias en cuanto a productividad, calidad del aceite, adaptación al cambio climático y la oferta de otros servicios ecosistémicos, por ejemplo la capacidad para el aumento y mantenimiento del secuestro de carbono (C) fijado en la biomasa y en el suelo. Proyecciones para Costa Rica anticipan que ante un escenario creciente de demanda energética, las fuentes tradicionales de energías limpias del país no podrán crecer más a partir del 2032 [1] [2].

Durante los últimos ocho años, un grupo interdisciplinario de investigadores de las universidades estatales de Costa Rica, han venido realizando estudios sistemáticos sobre el cultivo de la Jatropha curcas (Tempate) [3], para su uso comercial como fuente de aceite para producir biodiesel y a la vez iniciar el trabajo con otras especies con potencial bioenergético en Costa Rica como la higuerilla (Ricinus comunis) y el coyol (Acrocomia aculeata).

Cultivos con tempate, higuerilla y coyol, son una alternativa que se está estudiando a nivel mundial para la obtención de aceite para la producción de bioenergía [2] [3]. Como todo cultivo, la productividad está relacionada con el dominio de cuatro factores básicos y que guardan interrelación: genética, sitio, edad y manejo. De ahí la importancia y necesidad de un proceso de investigación que permita enfocarse en el desarrollo e innovación en la agrocadena y lograr aprovechar de una mejor forma los recursos, no solamente en campo de la bioenergía, sino otros usos como farmacéuticos, alimenticios y cosméticos [2] [4] [5] [6].

El objetivo de la propuesta fue evaluar sistemas de producción en cultivos bioenergéticos (Tempate (Jatropha curcas), higuerilla (Ricinus comunis), coyol (Acrocomia aculeata)) en tres sitios del país, como fuente de energías alternativas, que contribuyan al fomento del desarrollo rural y a la disminución del consumo de combustibles fósiles.

\section{Metodología}

Las investigaciones realizadas tienen que ver con ensayos de mejoramiento genético del tempate (Jatropha curcas), el rescate del coyol (Acrocomia aculeata) y con el establecimiento de diferentes arreglos con los cultivos de especies con potencial bioenergético (tempate, higuerilla y coyol), combinándolos con plantas que se utilizan para alimento humano y animal.

Al ser Jatropha curcas (tempate) una especie en donde se tienen avances relacionados con el manejo agronómico [3], se están haciendo ensayos en el Banco de Germoplasma, ubicado en la Estación Experimental Fabio Baudrit (La Garita de Alajuela. Costa Rica), conducentes al mejoramiento genético de esta especie, que busca la mayor producción de frutos y la obtención de individuos libres de ésteres de forbol, para ello se ha iniciado con la determinación de la antesis y características del polen que será de gran utilidad para los ensayos de mejoramiento genético.

Para la determinación de la antesis se hicieron observaciones en el Banco de Germoplasma de tempate ubicado en la Estación Experimental Fabio Baudrit (EEFB), las cuales iniciaron a las 5 de la mañana por varios días, se instalaron cámaras trampas para determinar dicha apertura y receptividad. Se programaron colectas de polen a diferentes horas y diferentes días en el mismo sitio, las anteras fueron llevadas al laboratorio de Biología Molecular de la EEFB, ahí se revisaron al microscopio a $40 \mathrm{X}$, las muestras fueron colectadas cada media hora hasta las 11 am. 


\section{Rescate de coyol (Acrocomia aculeata)}

Debido a que en Costa Rica no existe información relacionada con la biología reproductiva del coyol (A. aculeata) y a la necesidad de iniciar un proceso de domesticación con esta especie, se inició la colecta de germoplasma en la región del Pacífico Norte, Central y Sur y en el Valle Central, para establecer un banco de germoplasma en la Estación Experimental Fabio Baudrit Moreno (Alajuela, Costa Rica). Además para lograr su propagación se han realizado pruebas de germinación in vitro en el Laboratorio de Cultivo de Tejidos del Centro de Investigaciones en Biotecnología del Instituto Tecnológico de Costa Rica. Se inició con el establecimiento de un protocolo para la geminación in vitro de las semillas, se hicieron varias pruebas para determinar cuál era el explante más idóneo para sembrar. Se realizó cultivo de embriones y se tuvieron que hacer diferentes ensayos con medios de cultivo, para determinar cuál era el mejor. También se estandarizó el protocolo de desinfección de las semillas y los embriones in vitro. Actualmente se continúa trabajando en el proceso de aclimatación en el invernadero. Las pruebas posteriores corresponderán a la germinación de las semillas in vivo, según protocolos aprendidos en el Instituto Agronómico de Campinas, Brasil.

\section{Establecimiento de cultivos bioenergéticos}

El proyecto de investigación inició en el 2017, para el establecimiento de los cultivos con uso bionergético. Se contó con plantas de coyol (Acrocomia aculeata), como el cultivo principal, pero mientras se logra la obtención de la producción de frutos, que se estima que ocurre a los 5 años, se asoció este cultivo con higuerilla (Ricinus comunis) una oleaginosa que empieza su producción de frutos a los 8 meses aproximadamente y tempate (Jatropha curcas) otra oleaginosa que fructifica a los 2 años [3], de tal manera que los agricultores puedan contar con plantas para uso bioenergético, en diferentes momento durante el establecimiento de los cultivos. De forma paralela para obtener productos a corto plazo, se sembró maíz (Zea mayz) para alimento humano y pasto para alimento animal.

Con base en la importancia económica que tienen el tempate, coyol e higuerilla, se establecieron tres parcelas de 6000 metros cuadrados cada una, ubicadas en Santa Bárbara de Santa Cruz (Guanacaste), Valle Escondido en Palmira de Cañas (Guanacaste) y en la Estación Experimental Fabio Baudrit (La Garita, Alajuela), Costa Rica.

Las plantas empleadas para los ensayos fueron adquiridas de las siguientes fuentes: en el caso de coyol se obtuvieron plántulas de una edad de 3 años, ya establecidas en un vivero de la Estación Experimental Fabio Baudrit, semillas de tempate (accesión Comayagua), granos de maíz (variedad Diamantes 8043) y semillas de higuerilla de la variedad V9. Las semillas fueron sembradas en camas de germinación ubicadas en la Estación Experimental Fabio Baudrit, una vez establecidas las plántulas fueron llevadas a las parcelas experimentales. En el caso del pasto se utilizó el Clon Cuba 22.

Una vez preparado el terreno en cada uno de los sitios, se establecieron 5 tratamientos, los cuales consistieron en:

1. Coyol + Higuerilla

2. Coyol+ Tempate

3. Coyol + Pasto Cuba 22

4. Coyol + Cultivo anual (maíz)

5. Coyol (Control) 
Para cada uno de los tratamientos se establecieron 4 repeticiones. La unidad experimental de cada una fue de 300 metros cuadrados, 20 metros de largo por 15m de ancho, en un diseño irrestricto al azar de 20 parcelas. Cada una de las parcelas contenía 18 plantas de coyol, sembradas en una distribución triangular, con separación entre ellas de $5 \mathrm{~m}$. En el caso de los asocios de otros cultivos con el coyol, los mismos se ubicaron en las entrecalles de las filas de coyol. Para la higuerilla y el tempate, se estableció una fila de plantas entre las filas de coyol. La separación entre plantas de estos cultivos fue de $2 \mathrm{~m}$ una de otra. En total en cada parcela existen 3 filas del cultivo asociado, para un total de 30 plantas por parcela ya sea de higuerilla o de tempate.

En el caso de las parcelas con pasto o maíz, se estableció 3 filas del cultivo asociado en cada una de las 3 entrecalles por parcela. En el caso del pasto las distancias de siembra fueron de 1 por 1 metro entre plantas, para maíz, la distancia fue de $80 \mathrm{~cm}$ por $80 \mathrm{~cm}$.

El manejo de los cultivos ha estado basado en monitoreos de los mismos, contemplando las condiciones ambientales y del suelo, por lo que se cuenta con un protocolo de manejo agronómico. En cada uno de los sitios se tomó una muestra de suelo compuesta, que contempla el total del área donde se encuentran las parcelas. La muestra de suelo fue tomada en los primeros $25 \mathrm{~cm}$ de profundidad con un barreno. Fue cuarteada e identificada en cada caso. Las muestras fueron analizadas por el laboratorio de suelos del Centro de Investigaciones Agronómicas de la Universidad de Costa Rica.

Adicionalmente, se evaluó periódicamente el crecimiento de las plantas de coyol. Se realizó una evaluación inicial después del trasplante y posteriormente cada 6 meses se midieron las variables de respuesta como altura de la planta y diámetro de copa. La altura de la planta está dada desde la base de la planta hasta la punta de la hoja extendida más recientemente. El diámetro de copa se evaluó, contemplando la distancia entre los dos puntos opuestos de las puntas de las hojas más desarrolladas de las plantas. El tamaño de muestra fue de 5 plantas de coyol por parcela. Las evaluaciones fueron sistemáticas en el tiempo.

\section{Resultados y Discusión}

Se logró determinar que la receptividad del polen en tempate (J. curcas) se da de las 8 a las 10.30 am cuando el polen también presentó la mayor tasa de germinación. Se determinó que a partir de las 8:30 se empiezan a observar granos de polen germinados, la mayor cantidad se determinó entre 9:30 y 10:30 am (figura 1). Aspectos que se tomarán en cuenta en los programas de mejoramiento genético de esta especie.

Fotografía del ensayo de viabilidad del polen

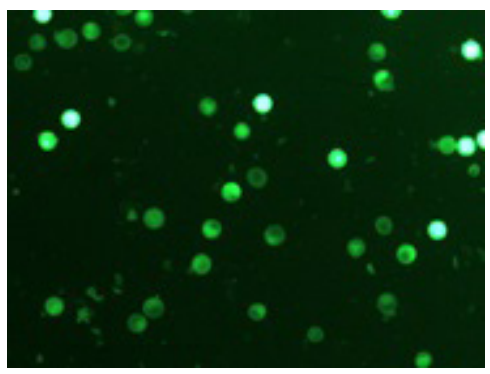

Fotografía del ensayo de germinación del polen

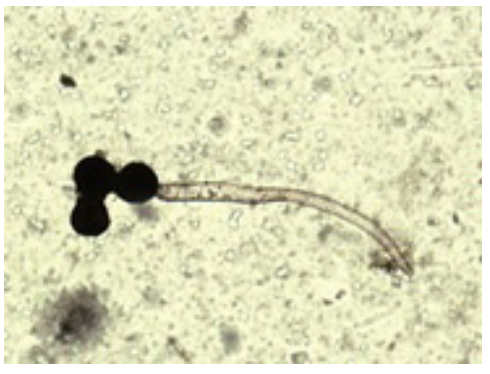

Figura 1. Viabilidad del polen y tubo polínico en granos de polen de tempate (J.curcas). 


\section{Rescate del coyol}

El coyol A. aculeata, tiene su centro de origen en Mesoamérica, desde el Sur de México (Chiapas) hasta el Noroeste de Costa Rica. Además en Suramérica en el norte de Colombia, el Sur de Brasil, Paraguay, Este de Bolivia y en el Norte de Argentina. También ha sido recolectada en la Isla de Santa Lucía en las Antillas Menores [7]. En Costa Rica además del Noroeste del país se han observado plantas en toda la vertiente del Pacifico. Según la clasificación de las zonas de vida de Holdrich, esta especie es posible encontrarla desde los bosques secos tropicales hasta Bosques muy húmedos premontanos [7]. Con base en lo anterior se ha colectado plántulas y frutos de coyol y ya se cuenta con un $77 \%$ de las accesiones de coyol (Acrocomia aculeata) colectadas de diferentes zonas del país ubicadas en el Banco de Germoplasma que se está estableciendo en la Estación Experimental Fabio Baudrit de la UCR, lo que permitirá realizar estudios relacionados con la biología reproductiva de esta especie, su conservación y la posibilidad de contar con material seleccionado para futuras plantaciones.

Para la multiplicación de esta especie, se cuenta con el protocolo de desinfección de las semillas de coyol (A. aculeata), se logró reducir la contaminación en un 100\%. Uno de los medios con los que se obtuvieron mejores resultados es utilizando ácido giberélico, como lo obtenido en otros ensayos similares [8] y carbón activado. La germinación de los embriones se da a partir de las 4 semanas de sembrados (figura 2). Se tiene un porcentaje de germinación del $90 \%$, utilizando semillas de frutos maduros. El desarrollo radical y foliar in vitro es rápido, por lo que a las 8 - 10 semanas se puede iniciar con el proceso de aclimatación.

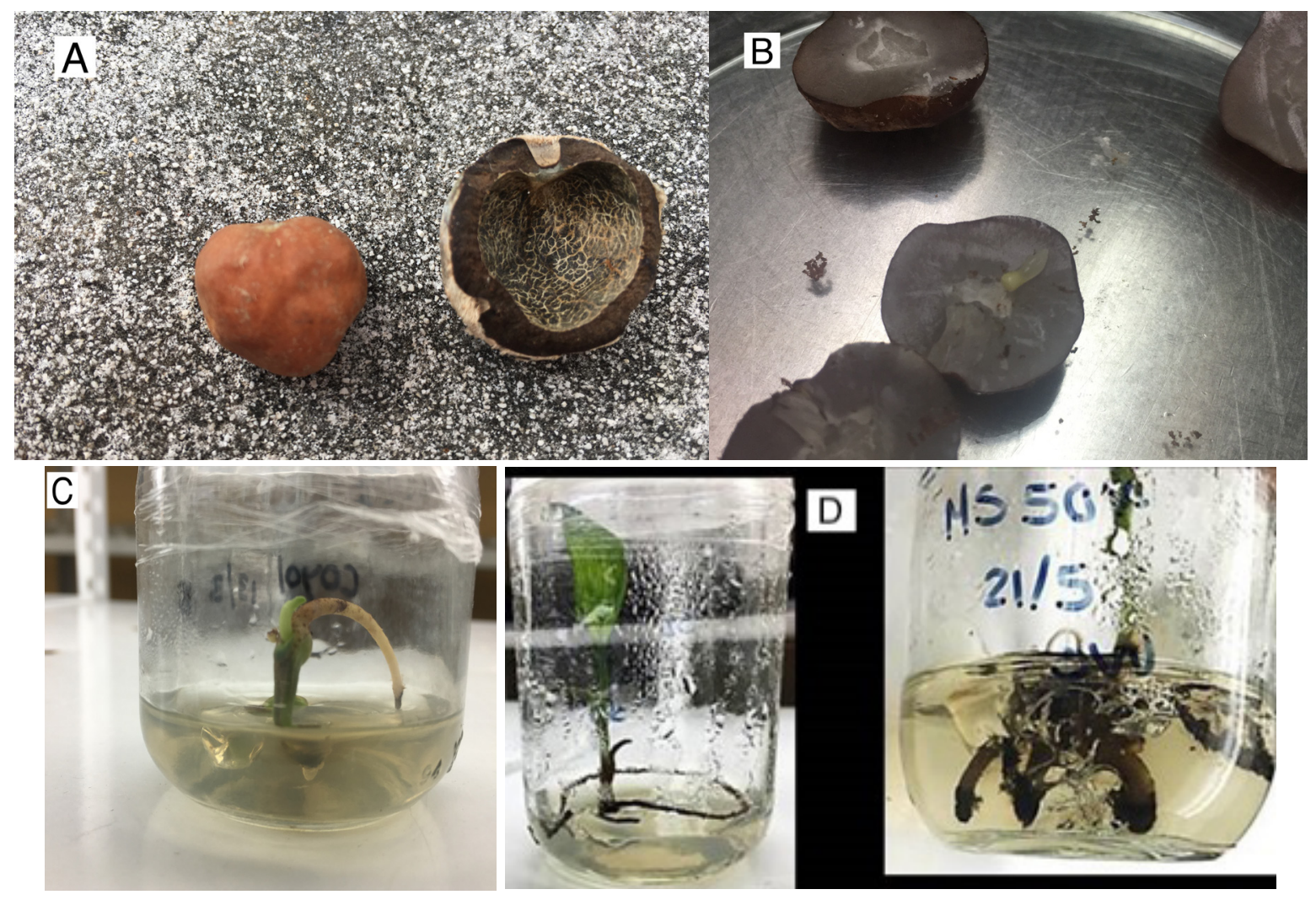

Figura 2. Proceso de introducción y germinación in vitro a partir de embriones de coyol. A, semillas maduras de coyol. B, separación y siembra de los embriones. C, embriones germinados. D, plántulas de coyol que presentan el mayor desarrollo radical. 


\section{Establecimiento de cultivos bioenergéticos:}

En las tres zonas donde se tienen los ensayos, los suelos tienen características químicas similares, valores de $\mathrm{pH}$ superiores a 5,5, niveles de calcio por arriba de $4 \mathrm{cmol}(+) / \mathrm{L}$, las otras bases se encuentran en niveles cercanas al nivel óptimo. En el caso del fósforo, los tres suelos tienen niveles menores a $10 \mathrm{mg} / \mathrm{L}$ (figura 3). Además los suelos son diferentes estructuralmente en cada sitio, factores que posiblemente están influyendo en el crecimiento y desarrollo de las plantas (figura 4).

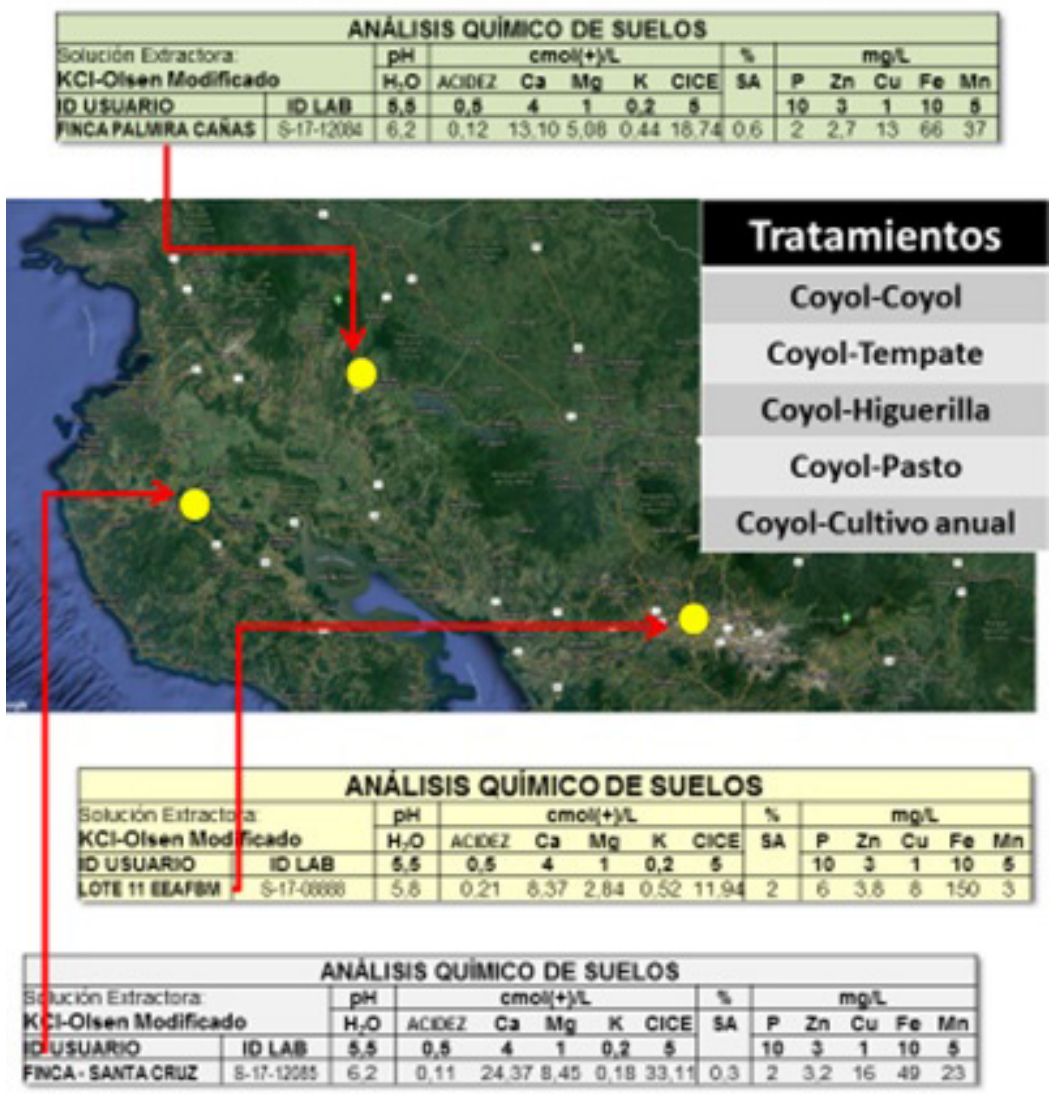

Figura 3. Ubicación y resultados de los análisis de suelo de los tres sitios donde se ubican las parcelas de cultivos bioenergéticos. Costa Rica.

Las plantas de coyol tenían una altura promedio de $90 \mathrm{~cm}$ al realizar el trasplante en septiembre de 2017. La altura de las plantas de coyol en los tratamientos en Santa Cruz, muestran que donde se asocia el coyol con el pasto y coyol en asocio con cultivo anual, tienen mayor altura con respecto al tratamiento control. Los tratamientos de asocio con tempate e higuerilla, tienen resultados similares al control. En el caso de la variable de respuesta diámetro de copa, los tratamientos donde se da el asocio del coyol con pasto y con los cultivos anuales también son los que tienen valores mayores al control (figura 4).

En Cañas, el comportamiento de la variable de respuesta altura, no tiene el mismo comportamiento al observado en Santa Cruz. Las plantas en general han crecido menos, en especial los tratamientos de los asocios de coyol con higuerilla, coyol con tempate y coyol con pasto. Las plantas de coyol en asocio con cultivo anual tienen una altura similar a las del 
tratamiento control. Un comportamiento similar se observa con los resultados del diámetro de copa (figura 4).

Los resultados en la EEAFBM, también muestran comportamientos diferentes con respecto a los otros dos sitios. Tanto en los resultados de altura de planta como diámetro de copa del coyol, en el tratamiento control muestra los valores más altos con respecto a los otros tratamientos. Es decir, en este sitio, las parcelas únicamente con coyol son las que presentan la mayor altura y diámetro de copa. Resalta en estos resultados, que el tratamiento coyol en asocio con pasto, muestra los valores más bajos de ambas variables de respuesta, esto difiere los datos obtenidos en Santa Cruz (figura 4).

\section{Santa Cruz}
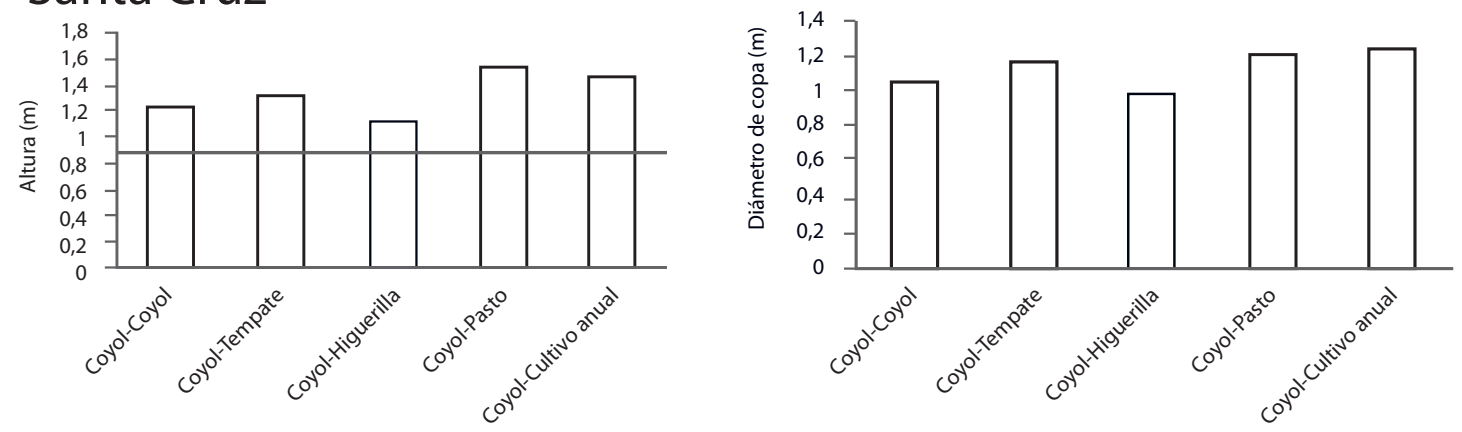

\section{Cañas}
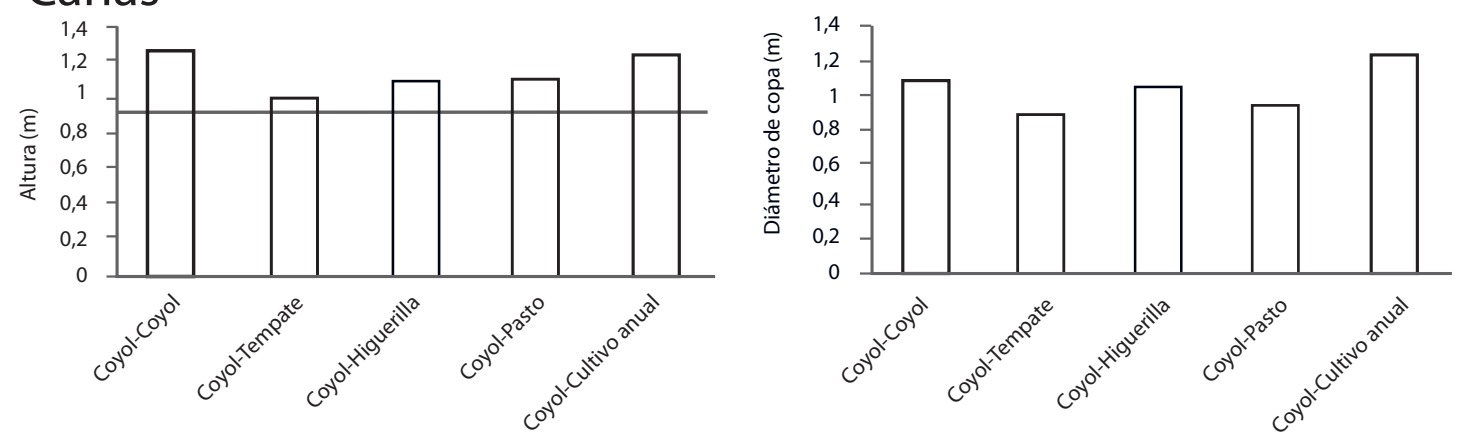

\section{EEAFBM}
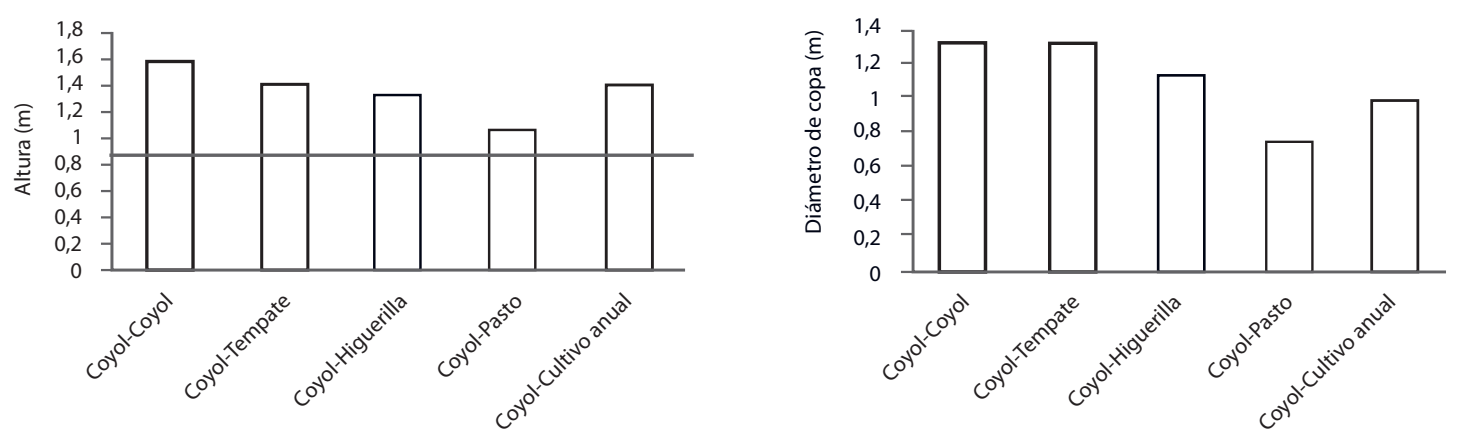

Figura 4. Desempeño de las plantas de coyol (A. aculeata), según el asocio y ubicación. Costa Rica. Septiembre 2017- septiembre 2018. 
Para llevar a cabo un análisis integral de estos resultados, es importante, observar los datos de la producción de pasto en los tres sitos. Datos obtenidos de cosechas cada 60-70 días, con una densidad de un metro entre cepas y 1.5 metros entre filas (figura 5).

Productividad potencial:

\section{2-72ton/Ha/Cosecha}

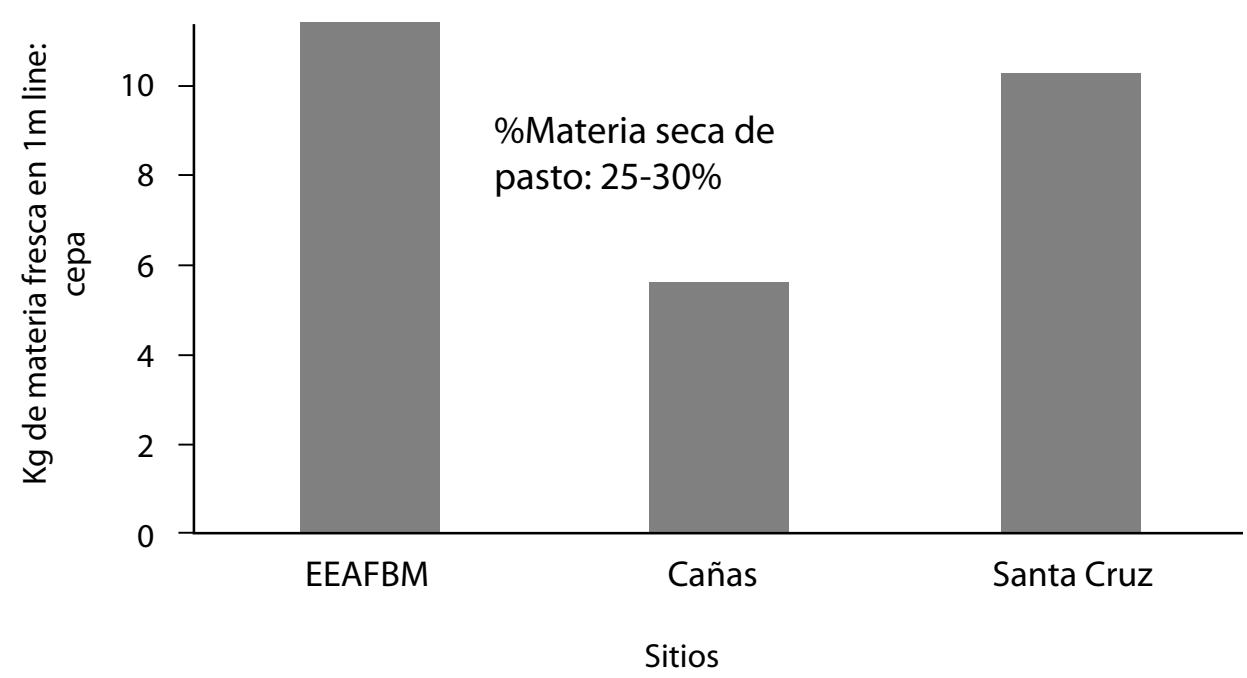

Figura 5. Rendimiento promedio de producción de biomasa del pasto Clon Cuba 22 en asocio con coyol (A. aculeata) en tres sitios. Costa Rica. Noviembre 2017-Septiembre 2018.

Tal y como se observa en la figura 5 con la producción de pasto ( $\mathrm{Kg}$ ) por metro lineal, la producción en la EEAFBM fue mayor en comparación a lo obtenido en Cañas y en Santa Cruz. La mayor producción de biomasa en la EEAFBM, podría estar influenciado en un sombreo sobre las plantas de coyol. Por lo tanto, se ve reflejado en un efecto sobre el crecimiento. Si bien este efecto puede ser valorado como no deseado para el coyol. Los rendimientos obtenidos en la producción del pasto cumplen con los objetivos del asocio, ya que obtener rendimientos de producción de biomasa de 62 a 72 toneladas/hectárea tres veces por año, se convierte en un ingreso atractivo para los productores, principalmente para los productores pecuarios de estas zonas. Por lo tanto, se podrían encontrar ajustes en las distancias de siembra entre el pasto y el coyol, para obtener buenos rendimientos de pasto y no tener interferencia de sombreo para el coyol.

\section{Conclusiones}

En Costa Rica se ha demostrado que hay sitios que pueden brindar niveles altos de productividad si se tiene control del material genético a utilizar y del manejo agronómico óptimo (paquete tecnológico del cultivo). Con el establecimiento de estos cultivos se espera ayudar a mitigar el efecto de las emisiones de gases de efecto invernadero causantes del cambio climático y contribuir a las metas que Costa Rica se ha propuesto para reducir la emisión de contaminantes al ambiente y mejorar la calidad de vida de la población costarricense. Así como reactivar la agroindustria para contribuir al impulso de opciones generadoras de empleo y riqueza [9]. Donde no solamente se obtendría energía por medio del biodiesel, sino subproductos a partir de la actividad agrícola e industrial. 


\section{Referencias}

[1] M. Carranza. "Utilización de la espectro radiometría para el estudio del estado nutricional y fisiológico en plántulas de Jatropha curcas L. (tempate)". Instituto Tecnológico de Costa Rica. Escuela de Ingeniería Forestal. Proyecto final de graduación para optar por el grado de Licenciatura en Ingeniería Forestal. Cartago, Costa Rica. 39 p. 2014.

[2] IICA. "Investigaciones en Jatropha alzan vuelo en América Latina y el Caribe. Innovación para cosechar prosperidad". IICA-Conexión Abril 2014. \#10. 2014.

[3] J. Loaiza, E. Arnáez, I. Moreira, F. Herrera, A. Ureña, J. Hernández. "Guía técnica para el establecimiento y producción del cultivo de Jatropha curcas (tempate) en Costa Rica". Editorial tecnológica. Costa Rica. 92 p. (ISBN 978-9977-66-244-2). 2012.

[4] C. Honaiser, R. Donomae, E. Sanjinez y C. Leite. "Diuretic and Anti-Inflammatory Activities of the Microencapsulated Acrocomia aculeata (Arecaceae) Oil on Wistar Rats". J Med Food 18 (6): 656-662. 2015.

[5] M. Lima, M. Mendes, P. Aiko, J.Braga, E. Machado. "Qualidade nutricional da polpa de bocaiúva Acrocomia aculeata (Jacq.) Lodd. Nutritional quality of the pulp of bocaiuva Acrocomia aculeata (Jacq.) Lodd.". Ciênc. Tecnol. Aliment., Campinas, 28(Supl.): 90-94, dez. 2008

[6] H. Makkar y K. Becker. "Jatropha curcas, a promising crop for the generation of biodiesel and value-added coproducts". European Journal of Lipid Science and Technology, 111(8): 773-787. 2009.

[7] N. Zamora, J. González y L. Poveda, Árboles y arbustos del bosque seco de Costa Rica, San Jóse, Costa Rica: Instituto Nacional de Biodiversidad, 1999.

[8] M. Viñas y V. Jiménez, «Factores que influyen en la embriogenésis somática in vitro de palmas (Arecaceae), » Colombiana de Biotecnología 13(1):229 - 242. 2011

[9] Programa Nacional de Biocombustibles. República de Costa Rica. Ministerio del Ambiente y Energía. Ministerio de Agricultura y Ganadería. 2008.

La presente investigación contó con financiamiento del CONICIT, FITTACORI, UCR y TEC. 